\title{
Intracerebral haemorrhage complicating dural arteriovenous fistula: a report of two cases
}

\author{
AE HARDING, B KENDALL, TJK LEONARD, MH JOHNSON \\ From the National Hospitals for Nervous Diseases, Queen Square and Maida Vale, London, UK
}

SUMMARY Two patients presented with unilateral peri-orbital pain, proptosis, chemosis and external ophthalmoplegia. They were shown to have dural arteriovenous fistulae related to the cavernous sinus. Intracerebral haemorrhage occurred in both patients within 18 months of presentation; this gave rise to focal seizures and signs of unilateral hemisphere dysfunction. The haematomas were in the region drained by the superficial middle cerebral vein ipsilateral to the shunt and are presumed to have been the result of locally raised venous pressure.

The majority of so-called spontaneous caroticocavernous fistulae are dural shunts between small meningeal branches of the external or internal carotid arteries and dural veins in the region of the cavernous sinus. The clinical syndrome associated with such fistulae comprises peri-orbital pain, tinnitus, proptosis, external ophthalmoplegia, dilated conjunctival and retinal veins, increased intraocular pressure, and ocular ischaemia. Dural arteriovenous malformations (or fistulae) communicating with the cavernous sinus are relatively rare and diagnosis is often delayed. In the two largest series of cases reported to date spontaneous closure of the fistula occurred in about $50 \%$ of patients, suggesting that the condition is often benign and self-limiting. ${ }^{2}$ In this paper we report the occurrence of intracerebral haemorrhage in two patients with dural arteriovenous fistulae; this potentially life threatening complication has not been well documented.

\section{Case reports}

Case 1 (NH B24694) This 63-year-old female was well until July 1981 when she suddenly developed sharp pain above the right eye radiating to the occiput whilst carrying a heavy suitcase. The pain was severe enough to prevent sleep for a few days but resolved gradually over three weeks. It recurred in the form of a constant dull retroorbital ache six to eight weeks later and in November 1981 the left eye was red and protruding. She also experienced high pitched pulsatile tinnitus in the left ear for two weeks around this time. In February 1982 the patient noticed

Address for reprint requests: Dr Harding, the National Hospital for Nervous Diseases, Queen Square, London WC1 3BG, UK.

Received 17 February 1984. Accepted 10 March 1984 diplopia on left lateral and upward gaze. This persisted until the time of her admission to the National Hospital in May 1982. There was no history of head injury.

On examination, apart from obesity, the abnormal findings were confined to the visual system. Visual acuity, fields, colour vision and the pupillary reactions were normal. On slit lamp examination the left eye was red with conjunctival chemosis and injection of the episcleral vessels. Intraocular pressure was $18 \mathrm{~mm} \mathrm{Hg}$ on the left and 13 $\mathrm{mm} \mathrm{Hg}$ on the right. There was a $2 \mathrm{~mm}$ left proptosis and ocular movements were slightly restricted on attempted elevation and abduction of the left eye. Diplopia occurred on laevoversion and laevoelevation. The retinal veins on the left were dilated but the optic discs were normal.

Routine haematological and biochemical investigations were normal, as were plain radiographs of the skull and orbits. A CT scan of the orbits showed enlarged extraocular muscles and dilatation of the superior orbital vein on the right. Angiography with selective injection of the internal and external carotid arteries revealed a dural arteriovenous connection related to the posterior half of the left cavernous sinus. This was supplied by the inferior hypophyseal branches of the right and left internal carotid arteries, and the middle and accessory meningeal arteries and the artery of the foramen rotundum on both sides. Drainage was through the left superficial middle cerebral vein over the posterior frontal convexity. The external carotid supply to the shunt was effectively embolised using lyophilised dura (fig 1).

Following embolisation the patient's dull retro-orbital pain resolved and she no longer noticed diplopia. On examination the afferent visual system was intact as before. Abduction and elevation of the left eye were normal but there was still a $2 \mathrm{~mm}$ proptosis. The left eye remained red but there was less chemosis. The fundi were unchanged. Intraocular pressure had risen to $27 \mathrm{~mm} \mathrm{Hg}$ on the left necessitating treatment with acetazolamide $125 \mathrm{mg}$ qds.

In August 1982 the patient had a transient episode of expressive dysphasia lasting 15 minutes. Her speech was 


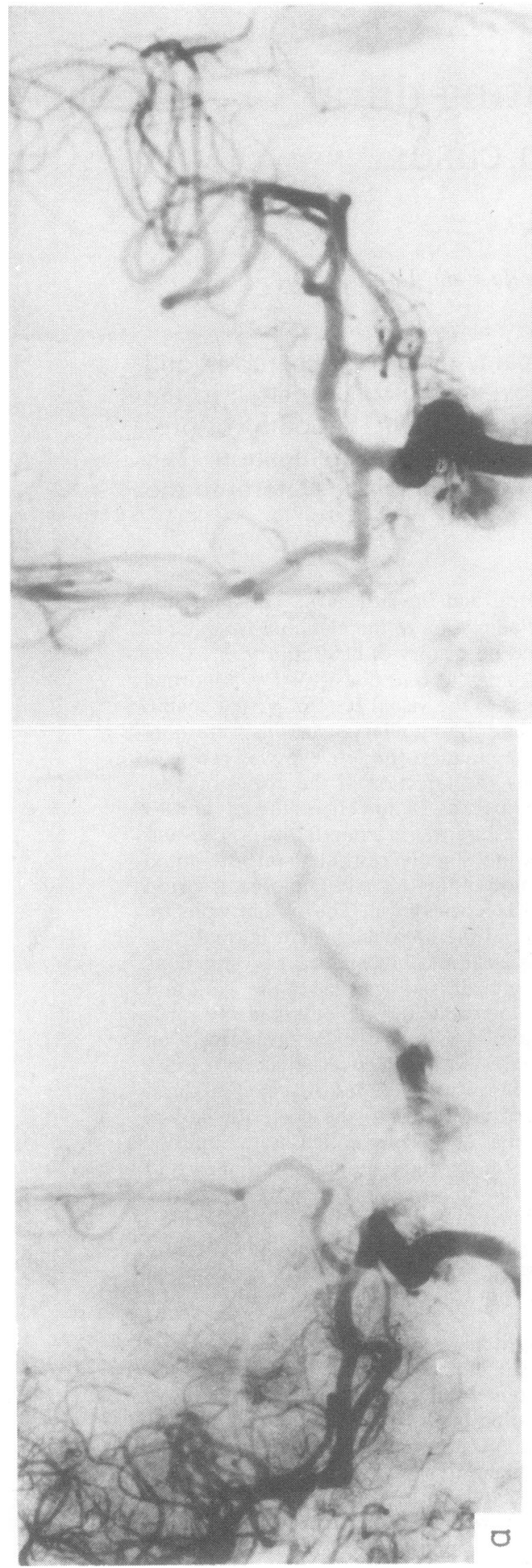

อ
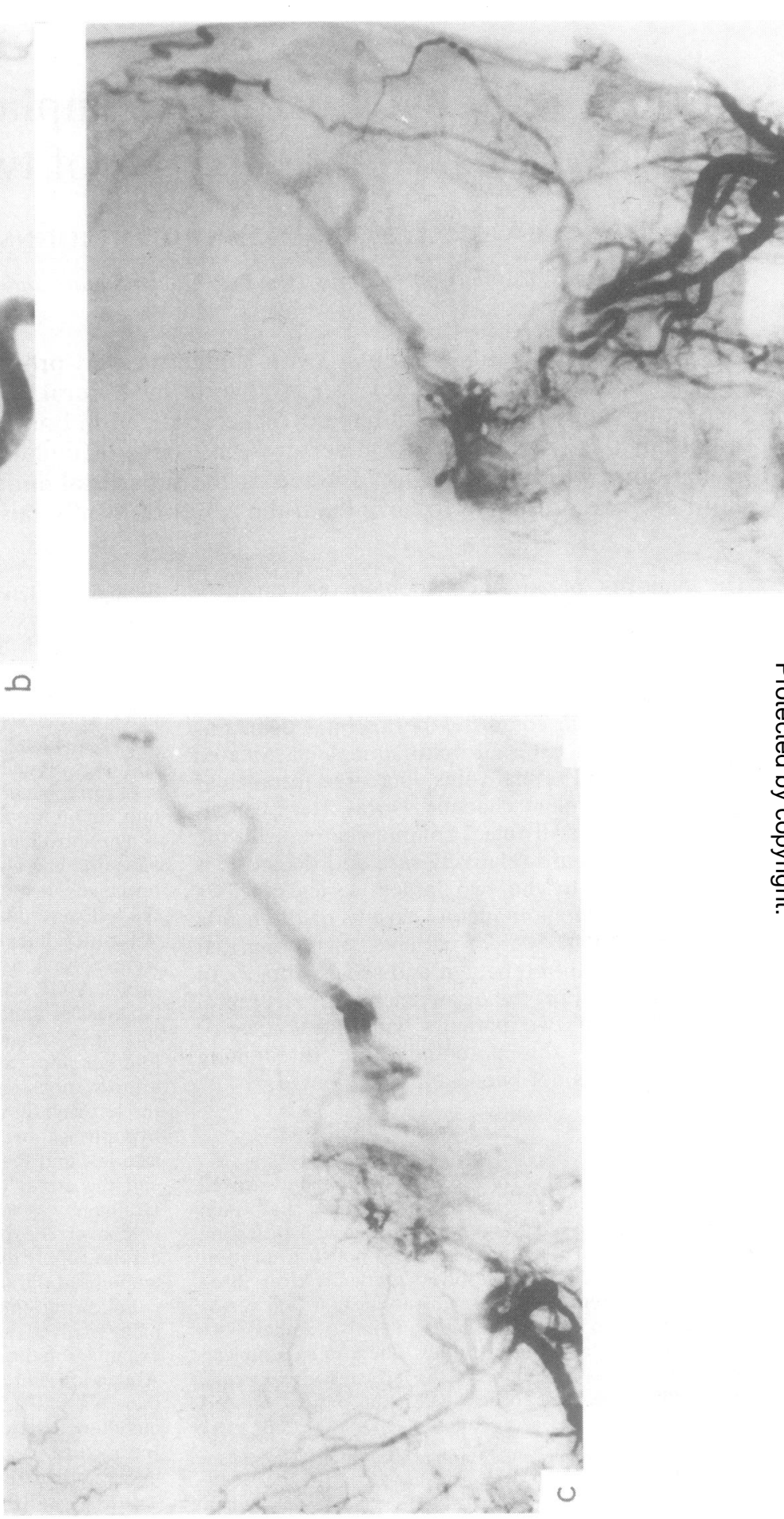

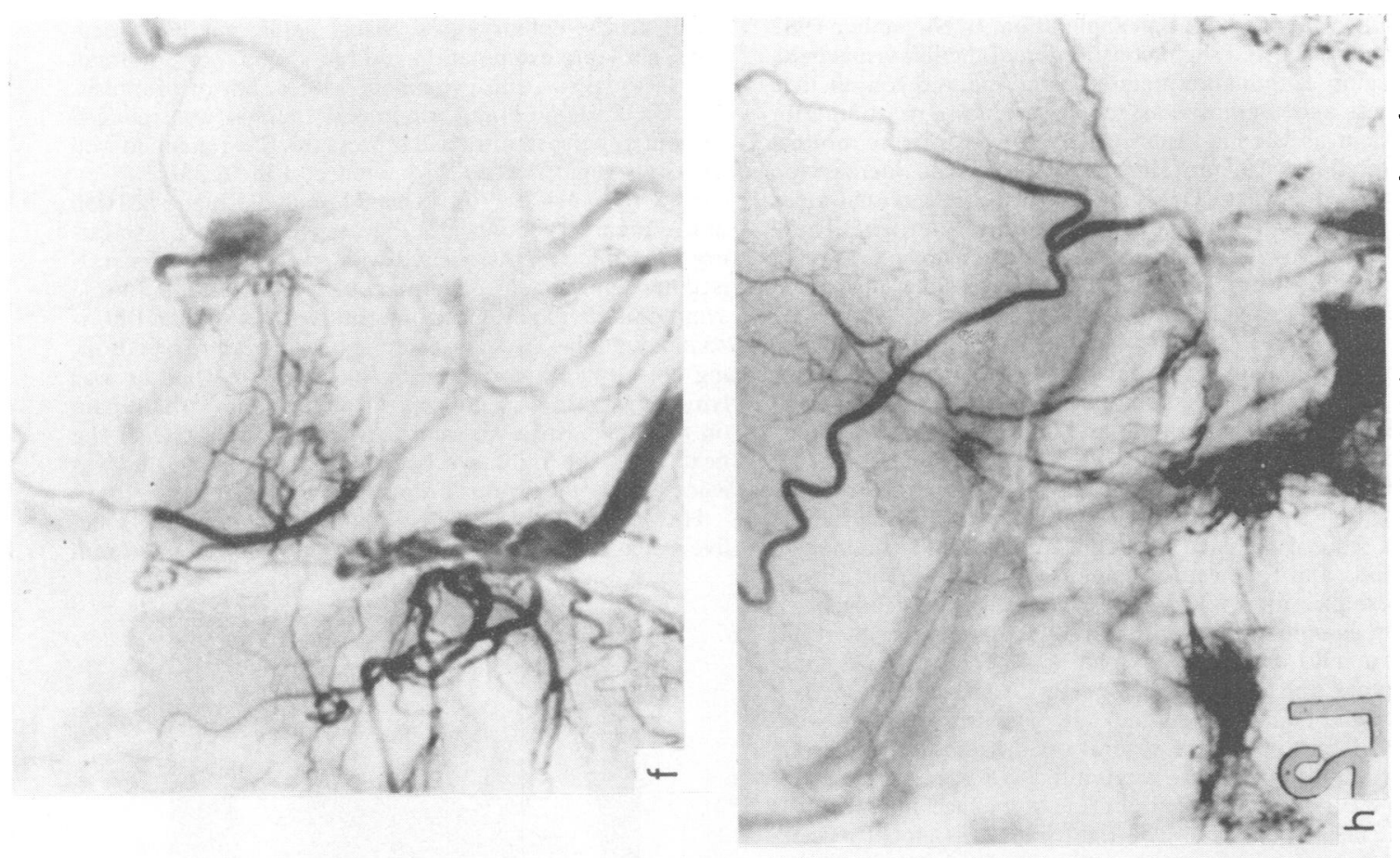

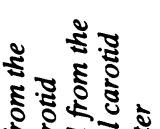
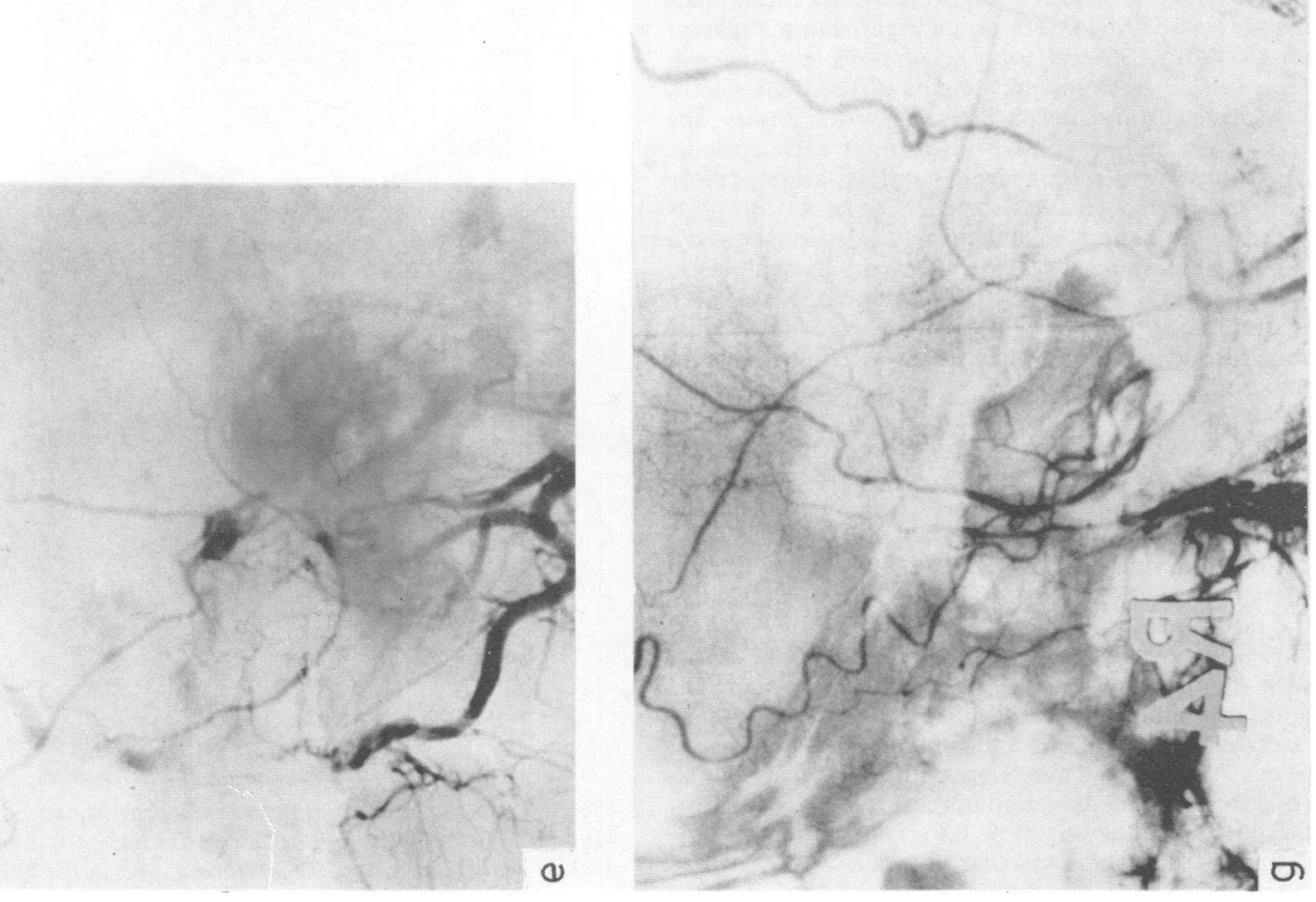

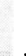

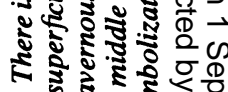

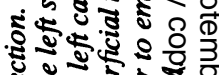

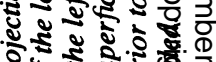
은

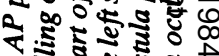

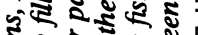
है 5 . क. 3 \% 5 : क्र

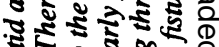
० 2 品 0 s. จ के ริำ

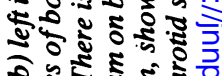

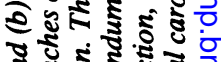

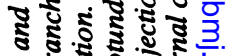
डิ ริ จำ

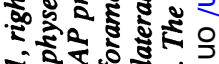
- करे क्ड ชै 2 50.

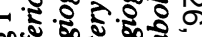
อำ 
normal when she was seen one week later and there were no other new findings on examination. In November 1982 perimetry showed slight constriction of the left visual field. Slit lamp examination demonstrated marked conjunctival redness and chemosis but there were no signs of anterior segment ischaemia. Intraocular pressures were normal bilaterally at $13 \mathrm{~mm} \mathrm{Hg}$ and there was no increase on attempted upgaze. On fundoscopy the left retinal veins were still relatively dilated compared to the right. The 2 $\mathrm{mm}$ left proptosis remained and abduction and elevation of the left eye were limited. Diplopia was only elicited on attempted laevoversion or laevoelevation.

In December 1982 her left retro-orbital pain recurred and was accompanied by diplopia on extreme upgaze. Two weeks later she noticed numbness in the thumb, index and middle fingers of the right hand on waking. Over the next three hours she developed weakness of the right hand, right facial numbness, and hesitancy of speech. During the afternoon she had frequent episodes of twitching of the right side of the face and right arm lasting for about 40 seconds and followed by increased weakness and speech disturbance for a few minutes. There was no headache.

On examination later that day the patient was alert but had a mild expressive dysphasia. There was a 4-5 mm proptosis and chemosis on the left. Visual acuity was $6 / 9$ on the right and $6 / 12$ on the left. The retinal veins were dilated and there were several small haemorrhages in the left fundus. Left upgaze was limited to $80 \%$. Pain appreciation was reduced on the right side of the face and there was right facial weakness. The right hand was globally weak, with loss of proprioception and abnormal two point discrimination. The tendon reflexes were symmetrical, apart from an increased finger jerk on the right, and the plantar responses flexor. Several focal seizures were witnessed. These consisted of clonic movements of the right side of the face and neck and flexion/extension of the fingers. She was conscious and responsive throughout each episode, which lasted about 30 seconds, but her dysphasia was more marked and her speech was slurred. There was no postictal increase in neurological deficit. A CT scan showed a small cortical and subcortical low convexity haematoma in the left precentral gyrus (fig 2). No abnormality was seen on bilateral carotid angiography with selective internal and external carotid injections. The fistula was assumed to have thrombosed.

The seizures were controlled with phenytoin and the patient's right sided weakness improved over the next five days but there was residual mild weakness and sensory loss in the right hand. Speech had returned to normal. The visual acuity on the left deteriorated to $6 / 36$, N18, with almost no colour vision. There was a left relative afferent pupillary defect. The left visual field was constricted with a central scotoma. Slit lamp examination showed gross chemosis and redness with a cellular reaction in the anterior chamber presumed to be of ischaemic origin. The intraocular pressures were $13 \mathrm{~mm} \mathrm{Hg}$ in both eyes on acetazolamide $125 \mathrm{mg}$ qds and timoptolol maleate $0.5 \%$ drops bd. There was a $5 \mathrm{~mm}$ left proptosis and slight limitation of elevation and abduction of the left eye. Fundoscopy on the left showed a slow flow retinopathy with multiple small haemorrhages and macular oedema.

In April 1983 the patient noticed improved vision on the left and resolution of her diplopia. On examination her visual acuity, colour vision, visual fields, pupillary reactions, slit lamp examination and fundoscopy were normal. The left eye was still prominent with $3 \mathrm{~mm}$ of proptosis. The acetazolamide and timoptolol therapy was reduced without any rise in intraocular pressure. She remained well with only slight residual prominence of the left eye.

Case 2 (NH MV99770) This 74-year-old man was well until January 1983 when he developed an upper respiratory tract infection associated with sweats, fever, generalised myalgia, and a productive cough. At the onset of these symptoms he experienced intermittent vertical and horizontal diplopia. Two days later he became aware of a droning noise "like a dynamo" in the right ear when he was lying down. He also noticed throbbing supra-orbital pain on the right which was most severe on waking. Over the next week the right eye became more painful; this was associated with obvious proptosis and ptosis.

He was admitted to the National Hospital, Maida Vale, five weeks after the onset of symptoms. There was a $7 \mathrm{~mm}$

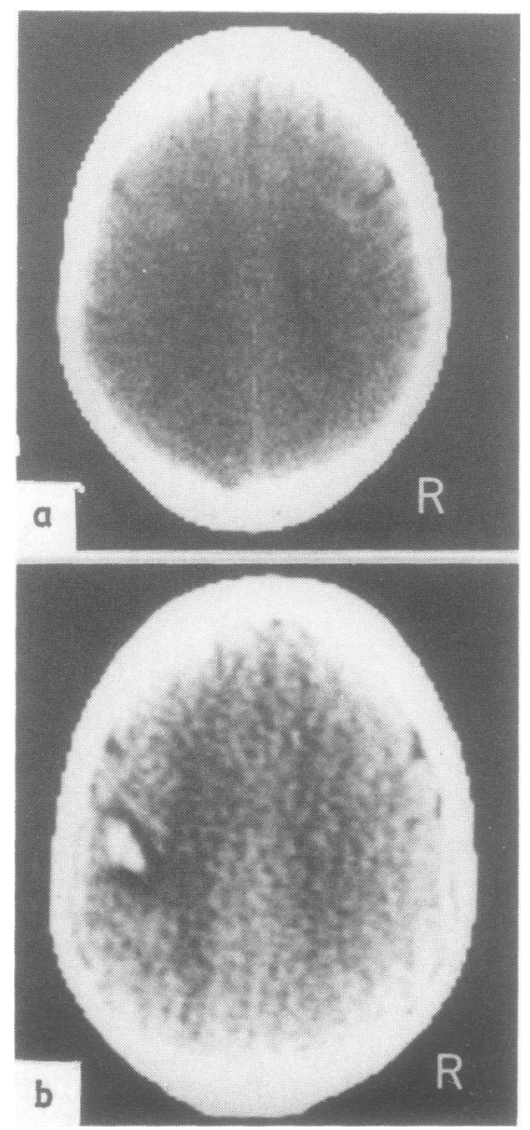

Fig 2 Case 1. A CT brain scan at the time of admission in May 1982 (a) was normal. In December 1982 (b) there was a small cortical and subcortical haematoma in the left precentral gyrus. 


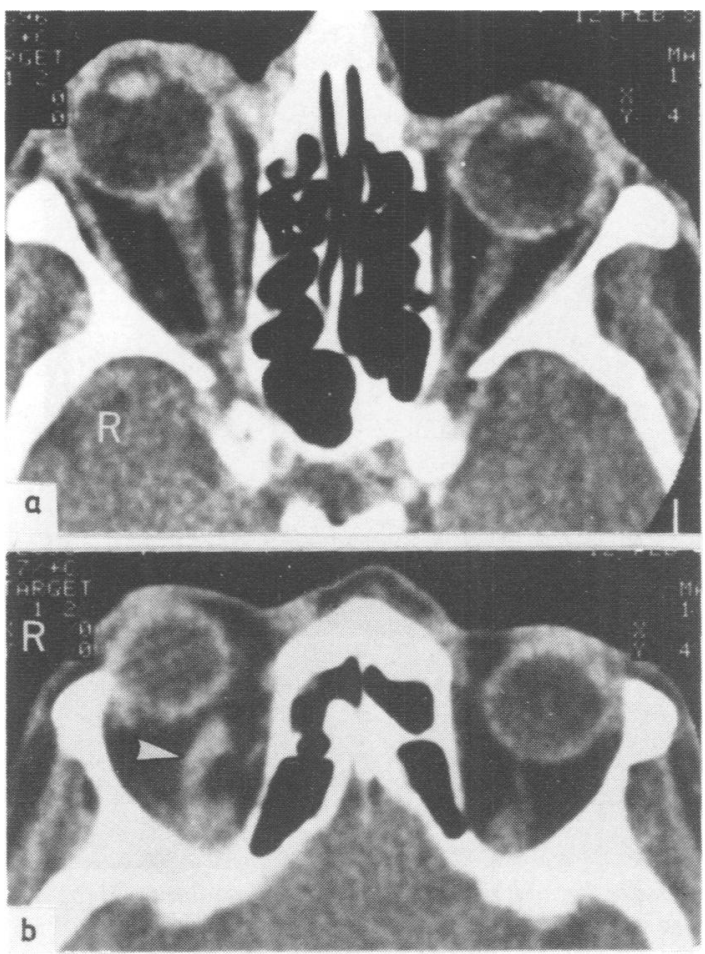

Fig 3 Case 2. CT scan of the orbits (a) in the plane of the optic nerves and (b) higher section. There is right proptosis, enlargement of the right external ocular muscles and optic nerve sheath, and thickening of the tissues adjacent to the inner canthus. The right superior orbital vein (arrow) is enlarged.

proptosis on the right with $5 \mathrm{~mm}$ deviation to the right, marked chemosis, conjunctival oedema, and ptosis. Visual acuity was $6 / 12$ on the right and $6 / 9$ on the left, with normal colour vision. The visual fields were full. Fundal examination showed mild bilateral venous congestion. There was a right relative afferent pupillary defect and a complete right external ophthalmoplegia. No orbital or cranial bruits were audible.

Routine investigations and plain radiographs were normal. CT scans (fig 3) confirmed the right proptosis. There was enlargement of the right external ocular muscles and optic nerve sheath. The right superior ophthalmic vein and other orbital vessels were enlarged and the tissues adjacent to the inner canthus were prominent due to dilatation of the angular vein. There was slight prominence of the right cavernous sinus. These appearances were entirely consistent with the presence of an arteriovenous fistula in the region of the right cavernous sinus. Cerebral scans were normal.

Over the next two weeks the patient's condition remained stable apart from the development of mild swelling of the right optic disc. A diagnosis of a dural arteriovenous fistula was made; it was decided to manage this conservatively in view of his age. The eyelids were sutured together temporarily in order to protect the cornea. Over the next two months the chemosis and conjunctival oedema improved. The ocular movements recovered to approximately $80 \%$ of normal in all directions. The visual acuity and fundal appearances did not change.

In early April the patient was woken one night by a rushing noise in the right ear. He then lost consciousness and was noted to have clonic jerking movements involving the left side of his body. When these ceased he regained consciousness but was unable to move his left arm or leg and his speech was slurred. His condition improved substantially over the next five days. He was then readmitted to the National Hospital.

On examination he was alert and had a moderately severe slurring dysarthria. He was normotensive. A high pitched bruit was audible over both orbits, louder on the right than the left. Visual acuity on the right was $6 / 12$. The visual fields were full. The right optic disc was slightly swollen and some small flame shaped haemorrhages were seen in the fundus, as well as venous engorgement. The chemosis and conjunctival oedema were not as severe as on previous examinations; there was a $4 \mathrm{~mm}$ right proptosis and moderate ptosis. Elevation and depression of the eye were limited to $45 \%$, and abduction to $80 \%$ of normal. There was a severe left facial weakness and mild weakness of the left arm. The tendon reflexes were brisker on the left but both plantar responses were flexor. Sensation was normal.

CT scan showed a haematoma in the right frontoparietal operculum with some surrounding white matter low density causing moderate mass effect (fig 4). The appearances of the orbit were unchanged compared with the previous scan.

The patient's dysarthria and hemiparesis improved over the next week. On review in September 1983 elevation of the right eye was reduced by $20 \%$ but other eye movements were full. There was a $3 \mathrm{~mm}$ right proptosis and mild chemosis. Visual acuity was $6 / 18$ on the right. The optic discs were normal but the retinal veins in the right fundus were tortuous and dilated.

\section{Discussion}

Both of these patients had the typical clinical features of dural arteriovenous fistulae in the region of the cavernous sinus. Most cases present with red eyes, pulsatile tinnitus, orbital pain, or diplopia. Mild proptosis, dilated conjunctival veins, restricted eye movements, and slightly raised intraocular pressure are the commonest findings on examination; audible bruits are rare. ${ }^{2}$ The diagnosis of a dural arteriovenous fistula was not proven angiographically in case 2 , but it was felt that there were sufficient clinical grounds for the diagnosis to be made confidently. Phelps and colleagues ${ }^{2}$ suggested that angiography should only be performed in this context if there is diagnostic uncertainty or if interventional therapy is contemplated.

The precise nature of these dural vascular abnor- 

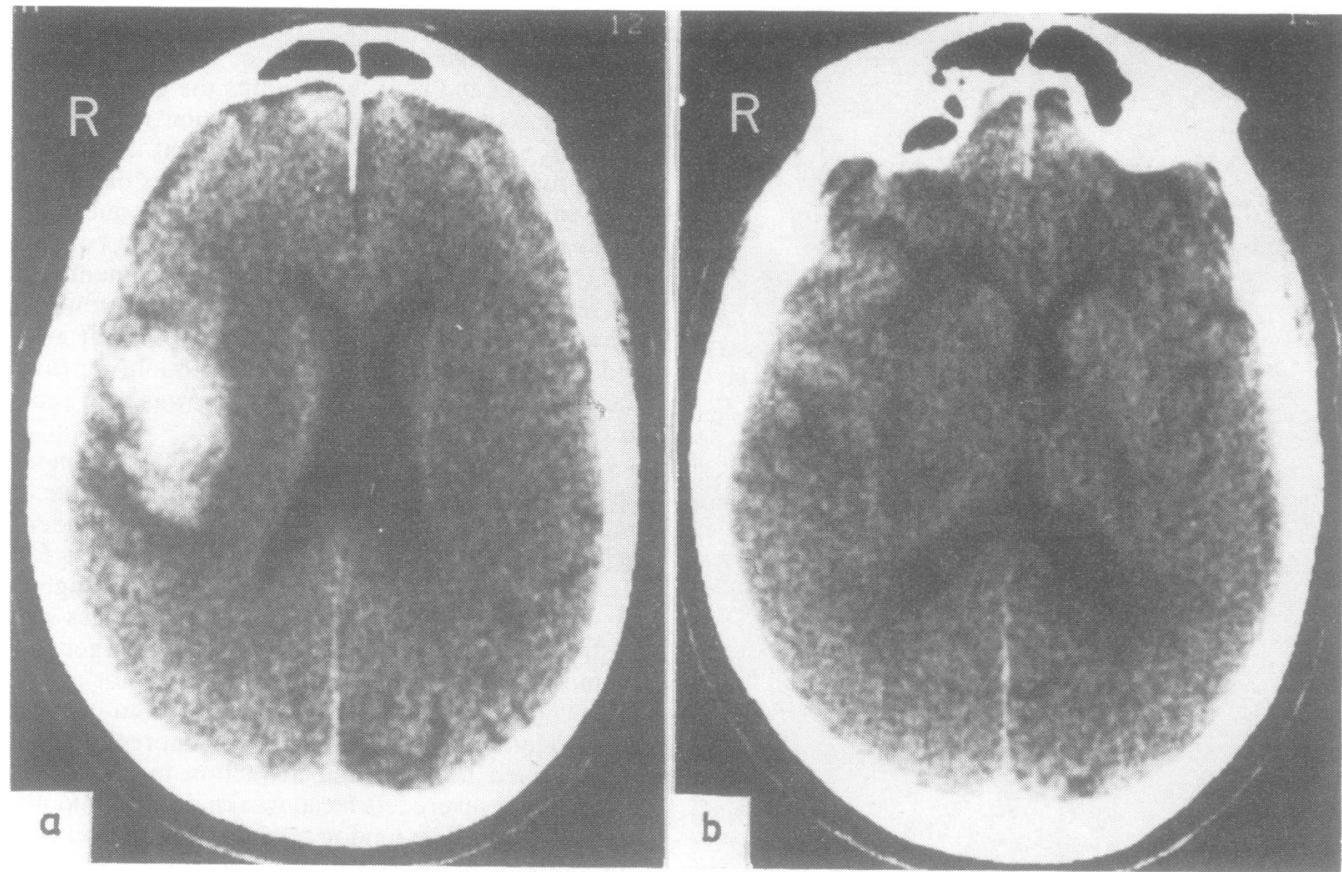

Fig 4 Case 2. CT brain scan, (a) section above the sylvian fissure through the frontoparietal operculum, and (b) lower section almost in the plane of the sylvian fissure. There is a haematoma in the right frontoparietal operculum with surrounding low density. It has sufficient mass effect to compress the right lateral ventricle and cause minor midline shift towards the left.

malities is uncertain. The frequent onset of symptoms in adult life, an increased incidence of diabetes amongst patients, and a preceding history of trauma or heavy lifting noted here in Case 1 , and in $30 \%$ to $50 \%$ of cases by other authors, ${ }^{2}$ suggest that they are acquired arteriovenous fistulae arising from rupture of thin walled arteries into veins which drain via the cavernous sinus.' Nevertheless it is impossible to exclude the presence of a congenital arteriovenous malformation in some patients. These would be indistinguishable radiologically from acquired fistulae which give rise to progressive dilatation of venous plexuses fed by arteries remote from the fistula. $^{3}$

Dural arteriovenous fistulae related to the wall of a cavernous sinus may drain through any or all of the veins which communicate with the sinus, namely the orbital veins passing through the superior orbital fissure, the superior and inferior petrosal sinuses draining to the jugular vein, the pterygoid venous plexus passing through the foramen ovale and foramen of Vesalius, veins traversing the foramen rotundum to the pterygopalatine fossa, and also through cortical veins which normally drain towards the cavernous sinus. The increased venous and decreased arterial pressures result in a reduction of blood flow, giving rise to the clinical manifestations of low capillary perfusion such as retinal haemorrhages and microaneurysms. ${ }^{4}$

Thrombosis often occurs in veins draining arteriovenous fistulae. It is especially frequent in venous sinuses and may cause diversion of venous drainage, such as the unusual flow into cortical veins as occurred in Case 1. Thrombosis can also precipitate cerebral venous infarction which is commonly haemorrhagic.

Intracranial haemorrhage is an unusual complication of dural arteriovenous fistulae. Subarachnoid and subdural haemorrhage occur sufficiently often to warrant considering a diagnosis of dural arteriovenous fistula in cases of unexplained extracerebral haemorrhage, but the shunts which bleed tend to be related to the superior sagittal, sigmoid, or transverse sinuses, drain through cortical veins, and rarely involve the cavernous sinus. ${ }^{3}$ Focal signs suggesting an associated intracerebral haemorrhage have been noted in such cases, ${ }^{5}$ although this was not confirmed by computerised tomography. The cases reported here are unique in that clinically typical dural arteriovenous fistulae related to the 
cavernous sinus were complicated by haematomas in the region drained by the superficial middle cerebral vein ipsilateral to the shunt. Bleeding was thought to be the consequence of locally raised venous pressure, possibly with thrombosis. Although both patients recovered following haemorrhage, this potentially serious complication should be taken into account when considering the relative merits of invasive or conservative therapy for dural fistula.

We thank Professor WI McDonald and Dr RW Ross Russell for helpful discussion and allowing us to report details of patients under their care.

\section{References}

' Newton TH, Hoyt WF. Dural arteriovenous shunts in the region of the cavernous sinus. Neuroradiology 1970; 1:71-81.

2 Phelps CD, Thompson HS, Ossoinig KC. The diagnosis and prognosis of atypical carotid-cavernous fistula (red-eyed shunt syndrome). Am J Ophthalmol 1982;93:423-36.

${ }^{3}$ Aminoff MJ. Vascular anomalies in the intracranial dura mater. Brain 1973;96:601-12.

${ }^{4}$ Sanders MD, Hoyt WF. Hypoxic ocular sequelae of carotid-cavernous fistulae; study of the causes of visual failure before and after neurosurgical treatment in a series of 25 cases. Br J Opthalmol 1969;53:82-97.

5 Djindian R, Merland JJ. Pure meningeal arteriovenous fistulae draining to cortical veins. In: Superselective Arteriography of the External Carotid Artery. Berlin: Springer Verlag, 1978:410. 